\title{
Stochastic rainfall fields time-series for probabilistic flood hazard assessment
}

\author{
Christine Poulard ${ }^{1, a}$, Etienne Leblois ${ }^{1}$ and Jean-Baptiste Faure ${ }^{1}$ \\ Irstea, Hydrology-Hydraulics Research Unit, Centre de Lyon-Villeurbanne, 5 rue de la Doua, BP 32108, 69616 Villeurbanne Cedex, France
}

\begin{abstract}
Probabilistic flood hazard assessment are usually carried out through juxtaposed reach-wise hydraulic simulations, using as input "representative" hydrographs for the studied return periods - at least by their peak discharge. However, reach-wise approaches have drawbacks, especially in the presence of natural or man-made singularities. An approach based on continuous simulation is developed to better assess flood hazard at the scale of the catchment and of the flood regime. A stochastic rainfall fields generator yields continuous times series, thus keeping the variability of the rainfall fields. Catchment-wise rainfall-runoff modelling, completed when necessary by a hydraulic model, allows to reproduce the individual and combined response of each feature to a heterogeneous rainfall event. The current CPU performances allow to process long rainfall time-series, but the codes have to be adapted to deal with unusually long input and output files. Local flood quantiles are then derived from discharge time-series, and flooding probability can be derived from local inundation frequency. This approach can be used in all contexts, urban floods or catchment-scale management; the modules have just to be chosen accordingly. This approach offers many perspectives, and in particular to better estimate local expected annual damages using damages time-series and multivariate damage curves.
\end{abstract}

\section{Flood Hazard Maps in the 2007 European Flood Directive... and beyond}

The majority of existing flood hazard maps were made for river overflow. Three main methods are used, sometimes in complementarity :

- using limits of historical floods (highest recorded water levels or limits of a selected historical flood) ;

- hydrogeomorphology, to deduce floodplain limits from geographical features ;

- hydraulic simulations, to estimate the extent of a flood generated by an input hydrograph built to be representative of a given return period.

Our communication deals with the latter, probabilistic flood hazard assessment of river overflow.

Directive 2007/60/EC on the assessment and management of flood risks of the European Union [1] hereafter referred to as Flood Directive or "FD", aims at "reducing the risk of adverse consequences of flooding". Member States must carry out a country-wise Preliminary Flood Risk Assessment, allowing to identify Areas with a Potential Significant Flood Risk (APSFR), on which must be subsequently drafted probabilistic flood hazard maps of 3 different return periods, flood risk maps and flood risk management plans (Articles 4, 6,7).

Flood hazard maps, and subsequent Flood risk maps, are key stages for the flood risk management process, by providing information crucial for decision-making. Point

\footnotetext{
a Corresponding author: christine.poulard@irstea.fr
}

12 of the preamble states: "In order to have available an effective tool for information, as well as a valuable basis for priority setting and further technical, financial and political decisions regarding flood risk management, it is necessary to provide for the establishing of flood hazard maps and flood risk maps showing the potential adverse consequences associated with different flood scenario,"

Article 13 sets the deadlines of the first cycle: the last documents of the process, the River Basin Management Plans, were due by the end of 2015. Article 14 sets the deadlines for the next update (dec. 2021) and states that updates will then be released "every six years thereafter".

The text does not provide technical guidance to make these maps ; however, point 16 of the preamble authorizes the use of existing documents and map to meet the requirements of the Flood Directive, and point 18 mentions that the assessments and maps must be based on 'best practice' and 'best available technologies', "not entailing excessive costs". Each country could therefore choose its own methodology, as long as it met the requirements of the FD (e.g. requirement for the probabilistic maps, see article 6). Now is a good time for feed-back analyses, cross-comparisons and proposals to improve flood hazard mapping, for the next cycle of the European Flood Directive, but not only.

This communication therefore briefly presents the most common approach to draft probabilistic flood maps, based on reach-wise modelling of hydrographs designed to be representative of a given return period. Its flaws 
will be pointed out, and our approach based on continuous rainfall-runoff simulations will then be presented. Significant improvements are expected for hazard diagnosis and project design. However, long continuous rainfall field time-series require adaptation to computation softwares and specific display tools.

\section{Reach-wise probabilistic modelling: how and why}

Producing maps with several return period, as required by the FD in the APSFR, allows to keep in mind there is no clear-cut limit: potential flood extent depends on flood severity. Besides, frequent and moderate floods can be useful for water and transportation infrastructure design, centennial floods are often used for land planning, and crisis management requires also information about extreme flood extent. Moreover, damage curves and population maps can be used to estimate the consequences associated to each probabilistic event, respectively in terms of economic damages or people affected. Thus, consequences per year on average can be deduced, offering a quantitative and objective measure of risk. Finally, the difference in annual average damages between a present state and an 'after-project" state (or several such scenarios) is a good measure of the annual average expected benefit of a project, to be used in costbenefit analyses (e.g. [2]).

Probabilistic assessments are therefore common (e.g. [3]), even more so now because of the European FD. This second part will identify the most frequent hypotheses behind the maps and question them.

\subsection{Hypothesis \#1: probabilistic hydrographs events}

To obtain a flood extent map representative of a given return period denoted $\mathrm{T}$, it appears logical to feed into a hydraulic model a hydrograph representative of $\mathrm{T}$. Its peak flow is obviously $\mathrm{Q}(\mathrm{T})$, the discharge quantile estimated for $\mathrm{T}$, obtained from statistical analysis of local hydrometric data, or by regionalization. But other characteristics must also be defined, like volume, duration and shape in general.

This method requires only one simulation of one event per return period to produce maps, which are furthermore immediately understandable as being the flood extent corresponding to a return period.

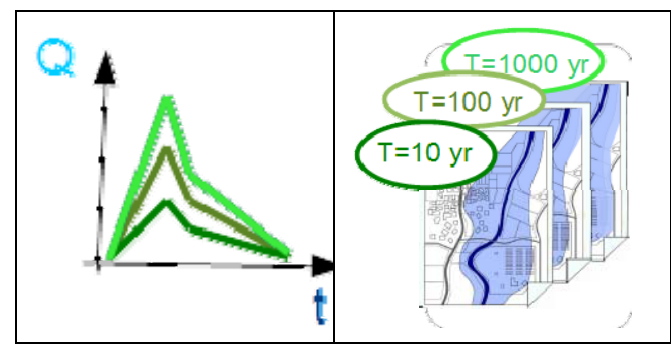

Figure 1. usual sequence for flood hazard mapping. First, hydrographs representative of return periods are built using information yielded by statistical analysis of discharge timeseries ; then, these hydrographs are fed into hydraulic models to produce maps

\subsection{Hypothesis \#2 : reach-wise approach}

The second underlying hypothesis is that the simulations are carried out on each reach independently. Indeed, the return period has only a local meaning. At each confluence, like in the example of Figure 2, it is not possible to add up the two upstream contributions: the resulting downstream flood would not remain of the same return period. Instead, the flood quantiles must be estimated again for this reach, a specific hydrograph must be designed, and an independent simulation must be carried out down to the next confluence.

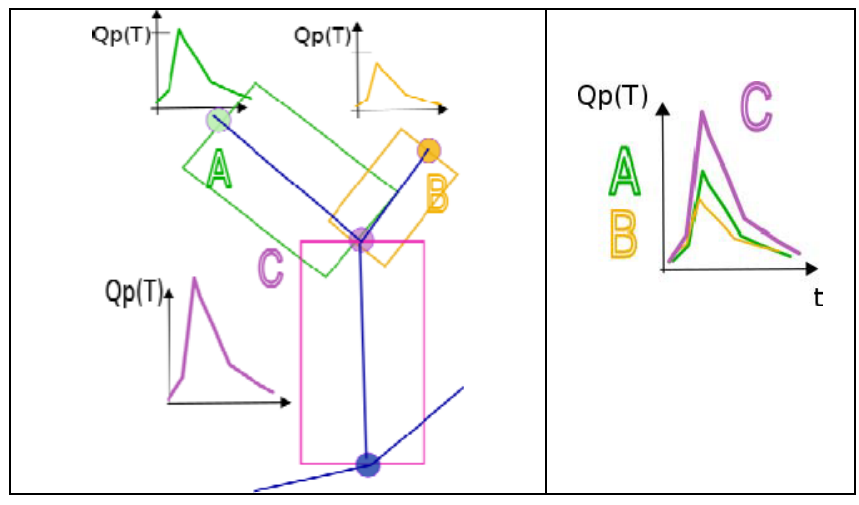

Figure 2. hydrographs are defined for each upstream node and routed independently in one stretch; they are not additive

This hypothesis is obliterated in almost every hazard maps, where procedures are applied to smoothe flood extent limits where they meet.

\subsection{Other hypotheses}

For all types of methods, other hypotheses have to be set and explicated: the limits of the studied domain (which tributaries are taken into account ...), and how the infrastructures are considered (neglected, correctly represented and assumed to have no risk of failure, or represented with breaches scenarios...). Dams and levees are not the only infrastructures that may modify the flood extent: sewerage system, road or railway embankments and bridges also have an impact on some floods.

\subsection{Limits of this approach}

The maps must be interpreted with precaution, due to the methods (uncertainty in flood quantiles, models reliability especially for large floods...), and to the underlying assumptions.

Working with "representative events" obliterates the variability of potential real events, which are by nature multivariate objects: many floods differing by their shapes and volumes could be labelled "centennial floods" at one given point, and yet gave different flood extents.

But the main flaw for hazard assessment is certainly due to the reach-wise reasoning, in particular in the presence of hydraulic infrastructures or geographical discontinuities (widening or narrowing floodplains...). Figure 3 illustrates what can happen during a real event: the rainfall is heterogeneous, and the efficiency of each 
hydraulic infrastructure depends on the local conditions: here, 3 mitigation dams are depicted, one of them will not have any effect because the local flows are low (1), the second works almost at its best, just before overflow (2), while heavy rainfall filled up the bowl of the last dam and caused overflow, so it will not really mitigate the peak discharge (3).

The flood in the upstream village (4) results of the floods upstream, including the effects of the dams. Local flooding may also be due to surface runoff (5), which is not often taken into account in modelling. Downstream flood (7) is the resultant of all these phenomena, plus the effect of a levee, and of the behaviour of the sewerage system, which might include overflows (6).

To correctly take into account the effect of the infrastructures at catchment scale, it is important to have local hydrometric data on a long enough period, capturing the combined effect of existing infrastructures ; if we want to model the flood extent of projected structures this information is not available.

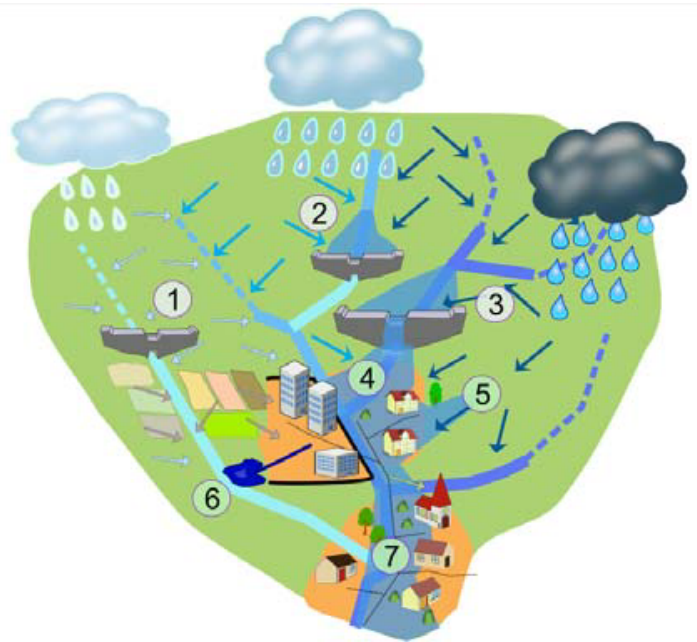

Figure 3. Possible individual and combined effects of hydraulic infrastructures during a flood event.

So, the reach-wise method is a common approach, easy to implement, that will yield correct results in the presence of only long-existing structures, which behaviour is correctly captured in local hydrometric data. In the other cases, with recent or projected structures, this method is flawed.

It is possible but complicated to try and estimate apriori correlations between contributions at confluences. Besides, To correctly take into account the effect of the infrastructures at catchment scale, it is important to have local hydrometric data on a long enough period, capturing the combined effect of existing infrastructures ; if we want to model the flood extent of projected structures this information is not available.

This is why we investigated another approach, based on rainfall-runoff modelling, to keep the upstreamdownstream dynamics.

\section{Towards a new approach based on rainfall-runoff modelling}

Rainfall-runoff modelling allows to work at the scale of the catchment, thus taking appropriately into account upstream structures, and to introduce spatial rainfall heterogeneity ([3 to 8]). On the other hand, it raises other issues, in particular how to define the rainfall scenarios, especially when one expects an estimation of the flood return period. Stochastic rainfall fields generator can provide required rainfall scenarios; we present here the method we are developing to obtain probabilistic maps.

\subsection{Stochastic rainfall scenarios}

The stochastic rainfall generator SAMPO offers the possibility to generate at will realistic rainfall fields at the scale of the catchment, provided the model is calibrated with rainfall records from several reliable gauging stations over a sufficient duration ([5], [6]).

Sampo is calibrated so as to produce rainfall fields with the same space and time geostatistical properties as the local climate, and validation is carried out by comparing rainfall quantiles calculated from stochastic series to their local estimates, at the time-steps relevant for the study. Weather types are estimated by clustering procedures during the calibration procedure, and variographs are calibrated for each of this weather types. The frequency of these weather types can be calibrated independently for each season.

\subsection{From rainfall fields to probabilistic flood extent}

In our first tests, we investigated how to use a set of events, issued from the stochastic generator, to improve the assessment of mitigation structures ([7], [8]). The probability of such stochastic events can not be set apriori, which anyway is impossible at the scale of the catchment, so their local return-periods were estimated aposteriori, by comparing the calculated discharges to the flood quantiles estimated from usual methods (statistical analysis of measured time-series or regionalisation).

In these previous works, rainfall-runoff modelling proved that the effect on flood hazard of hydraulic structures disseminated on a catchment was highly dependent on the space-and time specificities of the vent. However, using a limited set of events was a drawback, because the results were too dependent on the set of stochastic events, and so still a long way from real probabilistic assessment ([7]).

Computation time appeared less and less limiting, allowing to resort to larger sets of events, up to 4000 events assumed to represent 1000 years through the 4 largest events per year ([8]). Modelling so many events 
made it possible to assume that, at a given point, the quantiles could be derived from the modelled time-series. However, choosing a-priori independent events implied to define what is a significant rainfall event: implicitly, only the events significant at catchment scale and of a pre-defined durations were assumed to be of interest. Local heavy rains, that could cause local floods, were therefore excluded de facto. Building independent event also ruled out the study of successive events, between which storage facilities could not totally empty, and also posed the problem of fixing the initial condition, for moisture and storage level. It also prevented to include in the study the consequences of moderate floods, which of interest for instance regarding combined sewer overflows.

This is why the present study investigates the use of fully continuous rainfall fields time-series. The SAMPO software can provide very long series, but the question was to check if the rainfall-runoff modelling could handle them.

\section{Materials and methods}

Our approach implies a chain of models, each one providing an output file to be used as input file in the next module: the rainfall fields generator, a rainfall-runoff model, and a hydraulic model if water levels are required, or in the presence of hydraulic structures (Figure 4). This chain will be completed by a damage module.

Depending on the objective of the study, the rainfallrunoff module may be distributed or semi-distributed, and may be used to estimate the discharges along the network, or to generate input hydrographs for the hydraulic model.
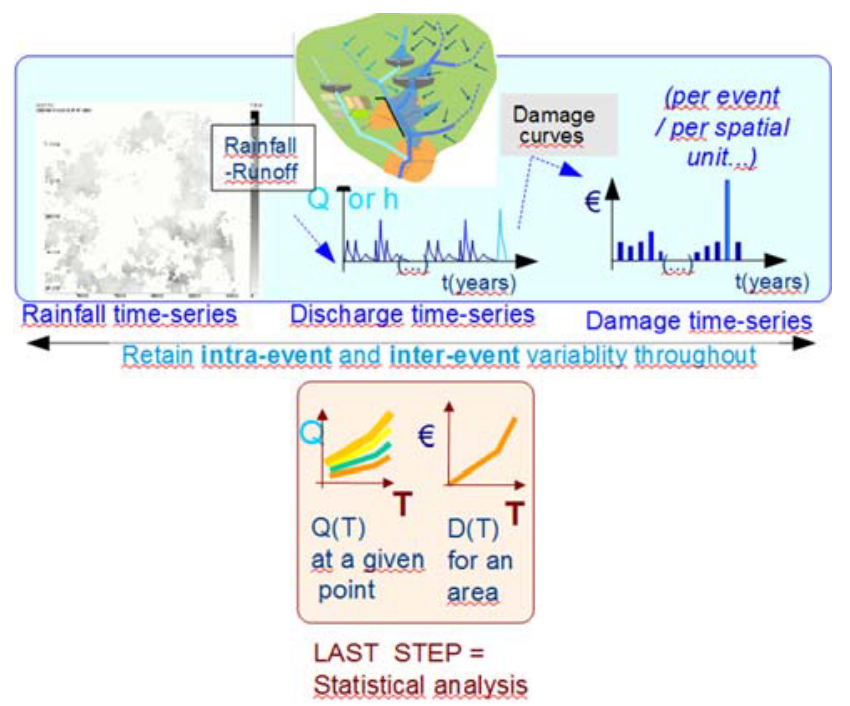

Figure 4. our approach for hazard and risk assessment (to be compared with figure 1).

\subsection{The catchment}

We chose a $144 \mathrm{~km}^{2}$ catchment near Lyon, where all necessary hydrometric and topographical data were available. Besides, the local authority in charge of floods has undertaken studies of 2 mitigation dams to protect the downstream part of the dam. So far, the studies consider the dams independently, for a given design flood. It is therefore an opportunity to test the potential of the method to assess the combined effect of the dams, over the whole hydrological regime. The current study is carried out for methodological developments, and its results are not yet suitable for design proposal ; to avoid over-interpretation of these results and in order not to interfere with ongoing studies, this catchment will be only denoted hereafter "Y144".

\subsection{Stochastic rainfall scenarios}

SAMPO was calibrated using about 40 rainfall gauges, located within or close to the catchment. Resolution of the rainfall fields were set as hourly in time, and $250 \times 250 \mathrm{~m}$ cells in space (figure 5).

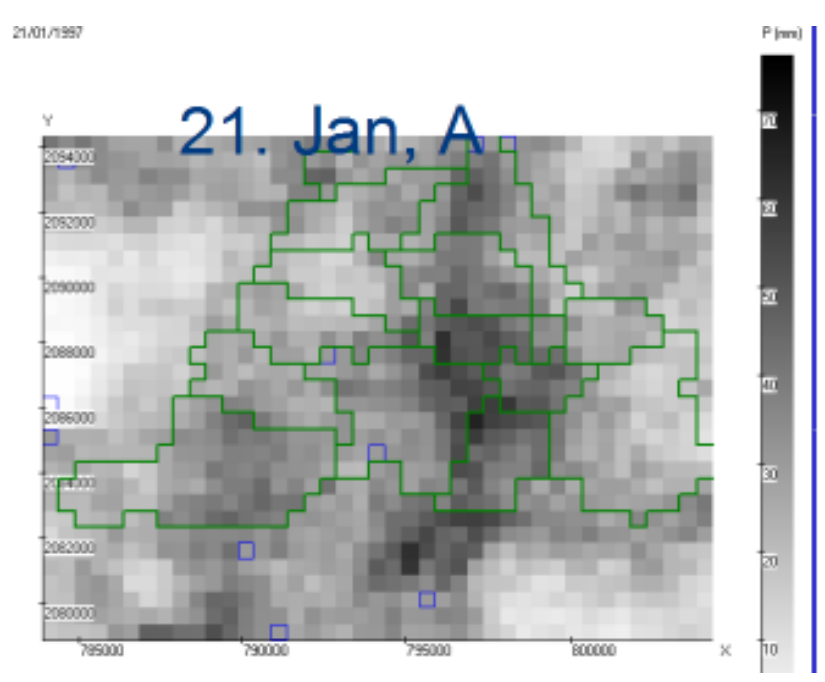

Figure 5. Example of rainfall field produced by SAMPO stochastic generator (blue squares indicate $r$ aingauges).

Having no a-priori estimation of the required duration of rainfall fields time-series, it was decided to produce a 1000-year time-series. The objective of the study was to test the efficiency of mitigation dams located on the main river (see figure 3), so the discharges are required mainly on this network. Therefore, a semi-distributed rainfallrunoff model was sufficient (see next paragraph). The code Hydrodem was used to delineate 59 elements with regard to catchment hydrology (figure 6). Sampo provided output files with the data required by the rainfall-runoff model, here one intensity value per hour averaged on each elements. So, the file issued from Sampo for this study contained "only" 518.256.000 values of rainfall intensity (59 elements x 24 hours x 366 days $\times 1000$ years). 
The rationale was to estimate the quantiles over the whole period and over shorter subperiods, to check the robustness of the method.

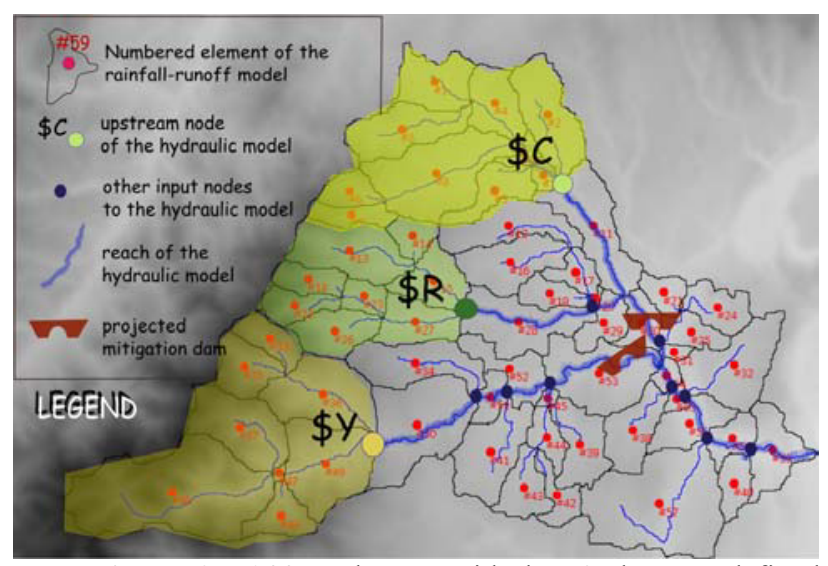

Figure 6. Y144 catchment, with the 59 elements defined for rainfall-runoff modelling and connections to the hydraulic model of the main rivers.

\subsection{Rainfall runoff module}

In our case, the discharges were not needed throughout the catchment but only along the network, and in particular reliable values are needed upstream the projected dams, and in the downstream floodplains where stakes exist.

Therefore, in the first place, a very simple transfer model (Sampo2Mage-Transfer, S2M-T) was used to generate input data into the hydraulic model and check how the hydraulic model could handle long input time-series at several nodes (see figure $6: 14$ point hydrographs and 18 lateral input). As a consequence, the output file has the same hourly time-step as the input rainfall data

For tests with 4000 events as generated by SAMPO ([8]), it was improved as $\mathrm{S} 2 \mathrm{M}-\mathrm{T}+$ to include a simple routing procedure between the elements.

\subsection{Hydraulic model}

Figure 6 presents the reaches where hydraulic modelling was needed, with the approximate location of the projected dams. The 1D model MAGE was used ([9]), which allows to model mitigation dams. In a first series of tests, a simplified cross-section geometry was used.

This model was written for events and short time-series (figure 7); this study is also an opportunity to test its compliance with long time-series and adapt it accordingly.

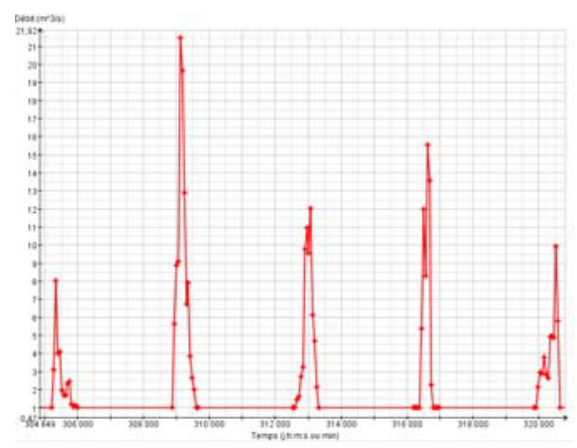

Figure 7. Display of input hydrographs at one node by the graphical interface of Mage : zoom on 5 events of a time-series constituted of 4000 juxtaposed 18-hour independent events (study [8])

\subsection{From hydraulic time-series to probabilistic maps}

After the water level time-series are estimated over the modelled domain, the last step for hazard assessment is to build the probabilistic maps. The procedure we follow for a given point is to count how many times the place is flooded during the simulation, and to deduce an experimental flood probability.

A map can be produced by carrying this estimation on every point, and maps for a given probability are obtained through defining thresholds.

\section{Results and Discussion}

The on-going tests confirm that the method is feasible and interesting, but identified technical problems that are being solved. We comment here intermediate results, final results will be available for the conference.

It appears that computational time is not an issue in itself; problems arise because softwares written for events or short time-series can not always cope with large input files : they load the whole input data and keep much of the computed variables in memory. This allows to display input data and output results as graphs (figure 7, 8 ), but computer memory can not deal this way with our much larger files. So, adaptations have to be made to handle large time-series, and specific data representation tools and syntheses have to be devised.

\subsection{Description of the rainfall data}

In the first tests ([8]), only 32 rainfall fields were used, so it was possible to display and analyze a-priori all rainfields maps. With 4000 events ([7]), it became necessary to identify a posteriori the events of interest. The problem is to capture simultaneously the space and time features in maps or graphs. 


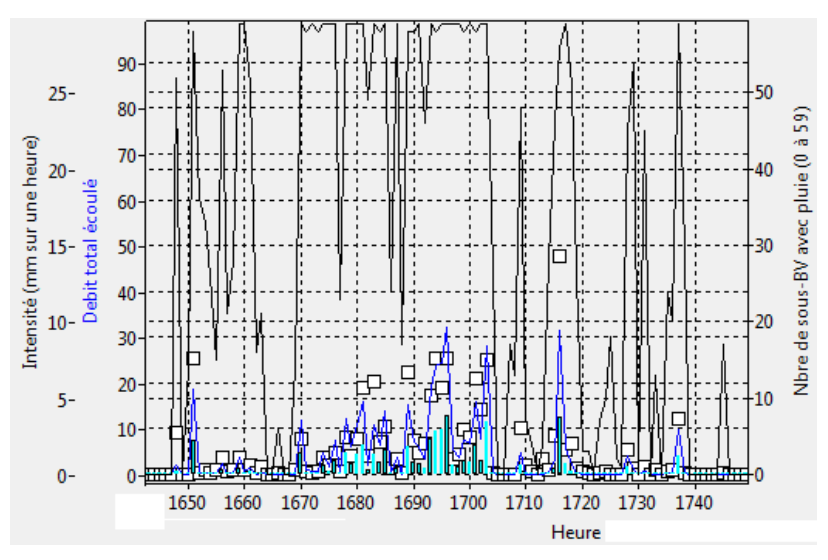

Figure 8. S2M-T graph of hourly rainfall intensity cumulated over the catchment (light blue histogram). The black squares correspond to the maximum intensity among the 59 elements, to illustrate the heterogeneity of the rainfall. The black line indicated the number of elements with non-zero rainfall intensity (right axis, between 0 and 59).

Figure 8 shows one of the graphs devised in S2M-T to display the average intensity for each time-step, to help identify the events susceptible of yielding the highest discharges. However, this graph can not be displayed for the whole time-series, because of memory limitations, so we have to chose a priori a limited sub-sequence.

It also displays the maximum intensity of one element and the number of elements with non-zero rainfall (right axis) to give a hint about spatial distribution. A box-plot of intensities on the 59 elements would give a better idea of the spatial variability, but could be intelligible only when zooming.

Figure 9 compares the annual maximum volumes over the whole catchment and in sub-regions. A zoom on year 558 shows that the annual maximum of the catchment (right) is very close to the sum of the annual maximum on 4 sub-regions (left). On the contrary, in the following year 504, the contribution of upper Y upstream the confluence and of the stretch downstream the confluence are only half or less of their own regional maximum.

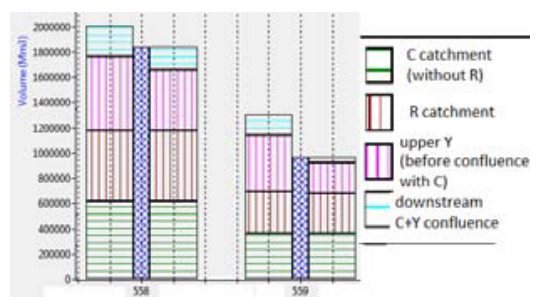

Figure 9. S2M-T graph of annual maximums of hourly rainfall volumes. Left stacked bar: annual maximum volumes for 4 regions, i.e. the catchments of the main tributaries and upper part of the main river, and of the lower catchment after both confluences. Central blue hatched bar: annual maximum over the whole catchment. Right stacked bar: contribution of the 4 regions to the catchment's annual maximum.

These examples illustrate the on-going efforts to devise graphs containing relevant descriptive information and yet remaining easy to interpret. Such representations can be used a-priori to identify potentially interesting events, or a-posteriori to analyze flood events after hydraulic simulations.

\subsection{Rainfall-runoff simulation}

$\mathrm{S} 2 \mathrm{M}-\mathrm{T}+$ used to load the whole input file with all the events, and to produce one hydrograph file per event ; however, it appeared unable to handle thus long continuous time-series. This is why another code, S2M-T, had to be written. In its current state, it is simpler (without transfer) and produce output files good enough to test feasibility of hydraulic modelling of our long timeseries. In parallel, a new code S2M-R suited to long timeseries is being developed to handle routing and varying soil moisture conditions, the latter being an important improvement allowed by continuous simulation.

\subsection{Input into hydraulic model}

S2M-T provides the hydraulic model with hydrographs for 3 upstream nodes, 10 other point hydrographs and 14 lateral input (figure 6). Even after removing redundant points, the size of the files describing point and lateral hydrographs for Mage is 670 Mo. It is not really a problem in terms of disk space, but Mage was not originally written to handle this amount of data. This is why the 1000-year time-series was split in 100 -year sub-series. Prior to any modification, each 100 yr time series took about 10 hour on a recent computer, with the possibility to run simultaneous simulation on multicores computers.

Any additional improvements of the rainfall-runoff model, like a finer time-step, and additional complexity, like introducing more projected structures, in particular in the upper parts of the subcatchments, will increase respectively the number and length of the hydrographs written as input files for the hydraulic model, thus enhancing the problems of computation time and output file size from hydraulic models.

So, these tests address algorithmic and files handling issues. Instead of starting by reading the whole input data, the information must be instead processed in streaming mode. Secondly, the procedures reading and writing files were carefully examined and modified: small improvements, which effect is totally negligible in usual projects, become here extremely important. Computation time has already been reduced thus down to 40 minutes. Another way of saving computation time is to reduce the amount of information in the output files, in terms of variables, time and space steps. These adjustments must be specified not only for our present test-case, but for future studies: we pointed out in the previous paragraph that the number and size of input hydrographs may be much larger in other studies, involving a larger catchment and/or more mitigation structures. 
Figure 9 and 10 displays first results, respectively annual maximums at the foot of one dam and after the confluence over a 100-yr simulation. However, a close examination of the time-series shows that the "mitigated" annual maximum sometimes happens for a different event than the reference annual maximum. Investigations are under way: some "events" are in fact a close succession of floods, which are less efficiently mitigated that isolated floods of comparable peak flood magnitude.

Thus, "hydrological" efficiency does not depend only on the peak discharge ; furthermore, damages also depend on the total submersion duration : continuous simulation allows to produce different event shapes and durations, to be accounted for into damage assessment.

The same tests will be carried out with other 100-yr time-series, to check for robustness, and with other dam scenarios (only one dam, other outlet dimensions...).

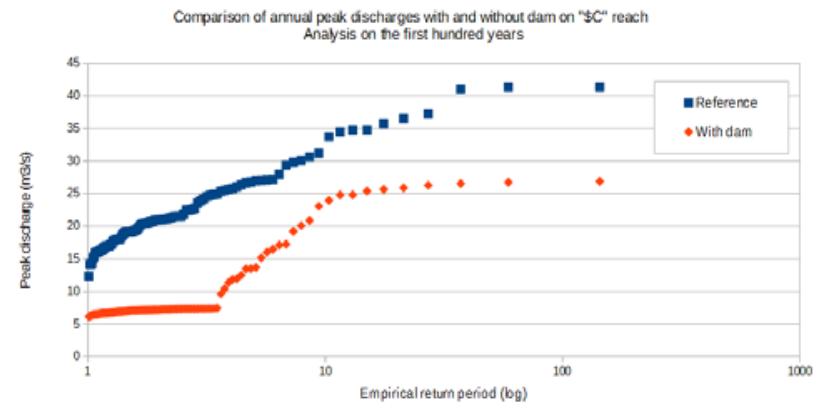

Figure 10. maximum annual discharges calculated by Mage over 100 years on the $\mathrm{C}$ reach downstream the projected dam.

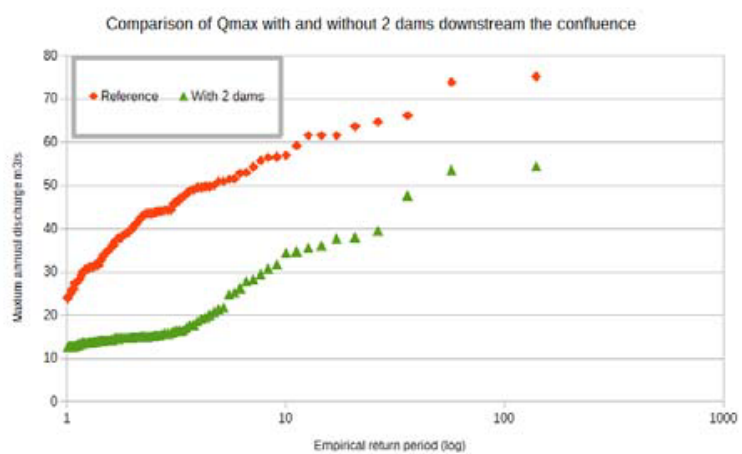

Figure 11. maximum annual discharges calculated by Mage over 100 years downstream the projected dams. In blue : natural discharges plotted against their empirical frequencies; in red : corresponding mitigated annual maxima in the presence of dams.

The Independent Subsections Method (ISM) developed by Proust et al. ([11]) is currently being implemented in Mage to improve the estimation of flow in the floodplain, by considering different 1D-flows with their specific headlosses in each subsection of compound channels (main channel and floodplains), and with mass and momentum exchanges at the interfaces between these subsections. Other authors are investigating continuous simulation approaches, using rather simplified 2D models ([10]). Coupling Mage with the full 2-D model Rubar is also feasible, because they share the same input files format and the same interface Pamhyr: Rubar could be called to simulate only the time steps with significant overflows.

\subsection{From hydraulic time-series to probabilistic maps}

Yearly or monthly maxima in each floodplain cell are sufficient to estimate flood frequency and make a hazard map.

However, the damages are not only linked to the peak discharge ([12], [13]): recent risk assessment procedures involve inundation duration, and may also involve velocity. One of the advantages of continuous simulation, compared to event-based simulations, is indeed to be able to account for the variability of hydrographs shapes, and of successive floods. So, the output files have to be written accordingly. One of the difficulties, to be treated according to economists' recommendations, is to merge successive events where relevant (a cell may be temporarily out of water and flooded again during a same event).

\section{Conclusions and perspectives}

Continuous simulation and/or heterogeneous rainfall fields are now well under investigation ([4], [6], $[14], \quad[15] \ldots)$. Continuous simulation does solve methodological problems, by allowing to take into account initial conditions (soil moisture and residual level of water in flood storage facilities), and by reproducing the real behavior of a set of flood mitigation structures under realistic heterogeneous rainfall events. It also offers opportunities for integrated management: along with flood descriptors, it could also estimate water resources (e.g. water stored in reservoirs), and Combined Sewer Overflows, with a hydraulic model including the sewerage system. However, many technical problems arise, simply because most of the existing codes are implicitly written to handle much smaller files. Thus adaptations to the codes are necessary to read, compute, write and plot relevant information. The size of the output files is not a problem regarding disk space, but regarding computing time and abilities of processing tools. Specific graphical interfaces are developed to handle these unusual files. Filtering output files as suggested above saves computing time, but the information lost might be considered useful later: the possibility to write complete files may not be totally overlooked, maybe as binary files, for traceability and future tests.

The logical next step is to build or adapt a risk assessment module, to turn time-series of distributed hydraulic variables into time-series of distributed damages. Their statistical analysis will yield distributed Expected Annual Damages, that can be used to produce maps and values aggregated over larger spatial units for decision-making, in particular to assess the economical relevance of flood risk management projects.

These risk assessment procedures will allow to improve the design a set of mitigating structures (choice 
of structures numbers and locations, outlet sizes...), using as objective function the reduction of average annual expected damages, or the cost/benefit ratio. Recent powerful computers and workstation allow to run many different such simulations, especially after computation and file-handling algorithms have been improved.

This approach could also include surface runoff assessment, and the effect of structures modifying this surface runoff before it reached the permanent rivers. This will require a finer distributed rainfall-runoff model, to the limit of the rainfall generator spatial resolution. Other on-going developments to coordinate local rainfall fields simulations at the regional scale, where different weather types can occur simultaneously, offer opportunities to use this approach at the scale of large catchments. Finally, it is also very interesting to try and include in our simulations estimated probabilities of levee breaches and dam failure to include these incidents into hazard and risk assessment.

\section{References}

1. European Union (2007). Directive 2007/60/EC on the assessment and management of flood risks. URL: http://eur-lex.europa.eu/legalcontent/EN/TXT/HTML/?uri=CELEX:32007L0060\& from $=\mathrm{EN}$

2. Saint-Geours N. et al. (2015). Ranking sources of uncertainty in flood damage modelling: A case study on the cost-benefit analysis of a flood mitigation project in the Orb Delta, France. Journal of Flood Risk Management 8(2): 161-176.

3. Environment Agency (GB): online flood maps, http://watermaps.environment-agency.gov.uk ).

4. Falter D. et al. (2015). Spatially coherent flood risk assessment based on long-term continuous simulation with a coupled model chain. Journal of Hydrology 524: 182-193.

5. Leblois E. and Creutin J. D. (2013). Space-time simulation of intermittent rainfall with prescribed advection field: Adaptation of the turning band method. Water Resources Research 49(6): 3375-3387.

6. Emmanuel I., et al. (2015). Influence of rainfall spatial variability on rainfall-runoff modelling: Benefit of a simulation approach? Journal of Hydrology.

7. Poulard C., Leblois E. and Faure J.B. (2014). Rainfall fields simulation to improve flood hazard and flood risk assessment. EGU General Assembly 2014. EGU. Vienna. (Austria), Geophysical Research Abstracts. 16: EGU2014-16146.

8. Poulard C. and Leblois E. (2009). Towards objective design of dry dams at watershed scale: how to take into account the spatial structure of the rainfall and its variability. Proceedings: hydrological extremes in small basins: 12th Biennal International Conference of the Euromediterranean Network of Experimental and Representative Basins (ERB), Krakow, Poland, 18-20 Sept.2008, IHP-VII, Technical Documents in
Hydrology, No 84, UNESCO, Paris, pp 21-28. URL : http://unesdoc.unesco.org/images/0018/001828/18284 6e.pdf.

9. Faure J. B. (2013). An Integrated Simulation Platform - PamHyr. Modeling Software. 5: 245-252.

10. Falter D., et al. (2013). Hydraulic model evaluation for large-scale flood risk assessments. Hydrological Processes 27(9): 1331-1340.

11. Proust S., et al. (2009). Nonuniform flow in compound channel: A 1-D method for assessing water level and discharge distribution. Water Resources Research 45(12).

12. Schröter K., et al. (2014). How useful are complex flood damage models? Water Resources Research 50(4): 3378-3395.

13. Schröter K., et al. (2014). Is probability of peak discharge a suitable proxy of damage in flood risk analysis? International Conference - Analysis and Management of Changing Risks for Natural Hazards. Padua, Italy: 7.

14. Moncoulon D. et al. (2014). Analysis of the French insurance market exposure to floods: a stochastic model combining river overflow and surface runoff. Nat. Hazards Earth Syst. Sci. 14(9): 2469-2485. URL : $\quad$ http://www.nat-hazards-earth-systsci.net/14/2469/2014.

15. Blanc J. et al. (2012). Enhanced efficiency of pluvial flood risk estimation in urban areas using spatialtemporal rainfall simulations. Journal of Flood Risk Management 5(2): 143-152. 